EPJ Web of Conferences 37, 01021 (2012)

DOI: $10.1051 /$ epjconf/20123701021

(C) Owned by the authors, published by EDP Sciences, 2012

\title{
Selected HERMES results on semi-inclusive meson production
}

\author{
Charlotte Van Hulse ${ }^{\mathrm{a}}$ on behalf of the HERMES Collaboration
}

Department of Theoretical Physics, University of the Basque Country UPV/EHU, 48080 Bilbao, Spain

\begin{abstract}
The HERMES experiment at DESY in Hamburg collected semi-inclusive deep-inelastic scattering data using the $27.6 \mathrm{GeV}$ HERA lepton beam and various gaseous light and nuclear targets. Results of charge-separated hadron-identified multiplicities and multiplicity ratios, measured on light and heavier targets, are shown as well as azimuthal moments measured from unpolarized hydrogen and deuterium targets. Furthermore, Sivers and Collins amplitudes, extracted from measurements on a transversely polarized hydrogen target, are presented.
\end{abstract}

\section{Introduction}

The HERMES experiment at DESY in Hamburg (Germany) has studied meson production in semiinclusive deep-inelastic scattering (DIS) for over a decade. In the experiment $27.6 \mathrm{GeV}$ leptons, i.e., electrons or positrons, were scattered off fixed gaseous hydrogen, deuterium, helium, or heavier targets, with hydrogen longitudinally or transversely polarized or unpolarized, deuterium and helium longitudinally polarized or unpolarized, and the heavier targets unpolarized. Lepton-hadron identification was performed by means of a transition-radiation detector, a preshower detector, and a calorimeter, with an efficiency exceeding $98 \%$ and a misidentification below $1 \%$. A dual-radiator ring-imaging Cherenkov detector allowed identification of pions, kaons, and protons, in the particle momentum range between $2 \mathrm{GeV}$ and $15 \mathrm{GeV}$.

Data collection on unpolarized targets provides a sample useful for the analysis of spin-independent quark fragmentation into final-state hadrons, and the usage of various types of (nuclear) targets makes the study of space-time evolution of hadron formation possible. In particular, multiplicities and multiplicity ratios were extracted from HERMES data in multi-dimensional kinematic bins, and are presented in sections 2 and 3, respectively.

Extending the analysis of semi-inclusive DIS data to the non-collinear distribution of hadrons, i.e., also taking into consideration their azimuthal distribution, provides access to the Collins fragmentation function [1], which describes the fragmentation of a transversely polarized quark into an unpolarized hadron. It enters the semi-inclusive DIS cross section in convolution with the Boer-Mulders distribution function [2]. The latter describes the distribution of transversely polarized quarks in an unpolarized nucleon. It is sensitive to the correlation between the intrinsic quark transverse momentum and its transverse spin. A non-zero Boer-Mulders distribution function provides strong evidence for non-zero quark orbital angular momentum. Results on the related experimental observables extracted from data collected on unpolarized hydrogen and deuterium are shown in section 4.

In the last section azimuthal asymmetries measured on a transversely polarized hydrogen target are presented. These provide access to the Sivers distribution function [3] and the transversity distribution function [4], entering the cross section in convolution with, respectively, the spin-independent and the Collins fragmentation function. The Sivers distribution function describes the spin-independent distribution of quarks in a transversely polarized nucleon, correlating quark intrinsic transverse momentum and transverse nucleon spin. A non-vanishing Sivers distribution function implies orbital angular mo-

\footnotetext{
a e-mail: cvhulse@mail.desy.de
}

This is an Open Access article distributed under the terms of the Creative Commons Attribution License 2.0, which permits unrestricted use, distribution, and reproduction in any medium, provided the original work is properly cited. 

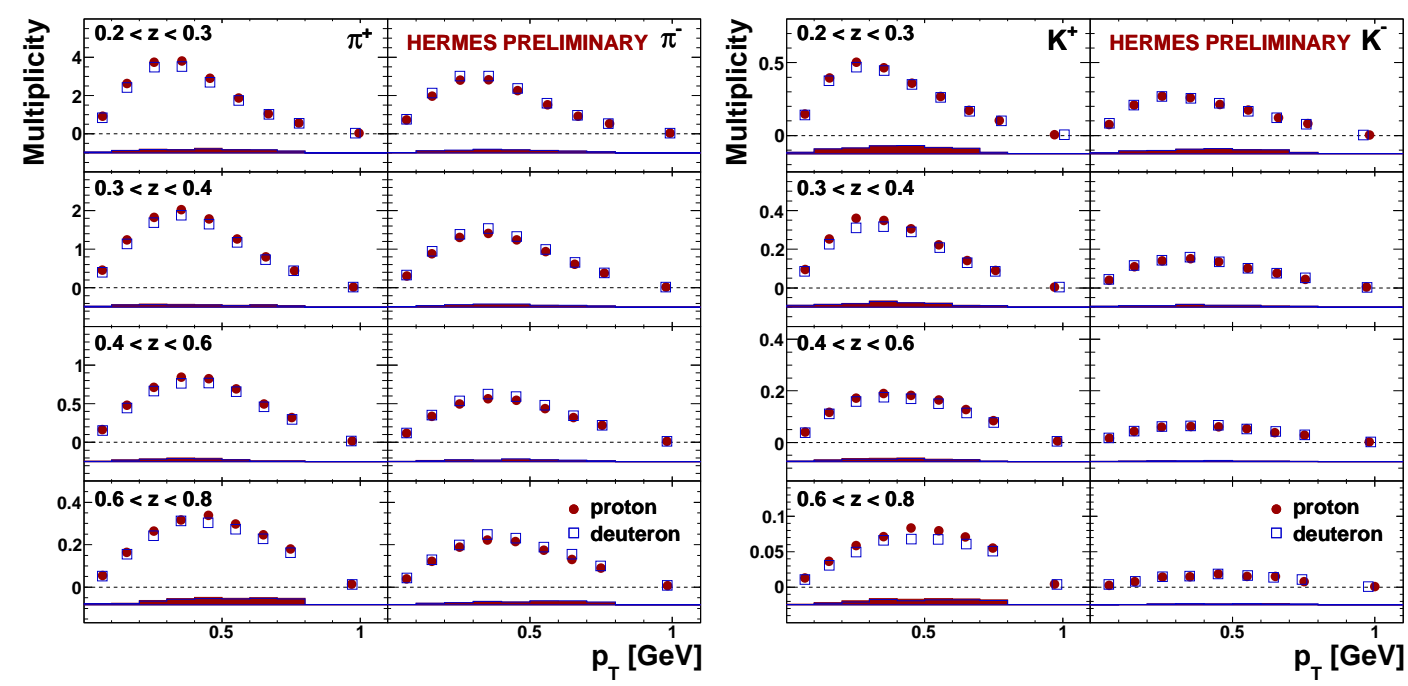

Fig. 1. Charge-separated pion (left panel) and kaon (right panel) multiplicities as a function of $P_{h \perp}$ (here labeled as $p_{T}$ ) and $z$ for protons (filled circles) and deuterons (open squares). Statistical uncertainties are represented by the error bars; systematic uncertainties are represented by the error bands.

tion of quarks [5]. The transversity distribution describes the distribution of transversely polarized quarks in a transversely polarized nucleon.

\section{Charge-separated pion and kaon multiplicities on hydrogen and deuterium}

Hadron multiplicities, i.e., hadron numbers per DIS event, were measured in three-dimensional bins in $\left(Q^{2}, z, P_{h \perp}\right)$ and in $\left(x, z, P_{h \perp}\right)$ for charge-separated pions and kaons, using an unpolarized hydrogen and deuterium target. Here $Q^{2}$ represents the negative squared four-momentum of the virtual photon that mediates the lepton-nucleon scattering, $z$ denotes in the target-rest frame the fractional hadron energy with respect to the virtual-photon energy, $P_{h \perp}$ the magnitude of the hadron momentum-component transverse to the virtual-photon three-momentum in the plane spanned by the three-momenta of the virtual photon and hadron (hadron-production plane), and $x$ the $x$-Bjorken variable. The experimentally extracted multiplicities are corrected for QED radiative effects, limited geometric and kinematic acceptance of the HERMES spectrometer, and finite detector resolution using an unfolding procedure, based on a LEPTO and JETSET Monte-Carlo simulation, similar to the procedure described in Ref. [6]. These obtained pion and kaon "Born" multiplicities for protons (filled circles) and deuterons (open squares) are presented in figure 1, as a function of $P_{h \perp}$ for different intervals in $z$.

As can be seen in figure $1, \pi^{+}$multiplicities for protons are larger than for deuterons, whereas for $\pi^{-}$the opposite is true. In addition, the ratio of the $\pi^{+}$and $\pi^{-}$multiplicities on proton (deuteron) ranges from $1.2(1.1)$, in the first $z$ bin, to $2.6(1.8)$, in the last $z$ bin. These observations can be understood by the dominance of scattering off a $u$-quark and a subsequent favored fragmentation of a $u$-quark into a $\pi^{+}$over an unfavored fragmentation of a $u$-quark into a $\pi^{-}$, the former pion containing a $u$-quark as its valence quark, whereas the latter does not. Similarly, the higher $\pi^{-}$multiplicities for deuterons in comparison to protons reflect the increased $d$-quark content in deuterons in conjunction with a favored $d$-quark to $\pi^{-}$fragmentation. For kaons an analogous behavior is observed in the comparison of the multiplicity ratio of both kaon types and in the comparison of $\mathrm{K}^{+}$multiplicities for protons and deuterons. However, the multiplicities of $K^{-}$seem to be insensitive to the target type. These results reflect the fact that $K^{-}$, being composed of valence quark types present in the nucleon only as sea quarks, can not be produced through favored fragmentation of nucleon valence quarks. 

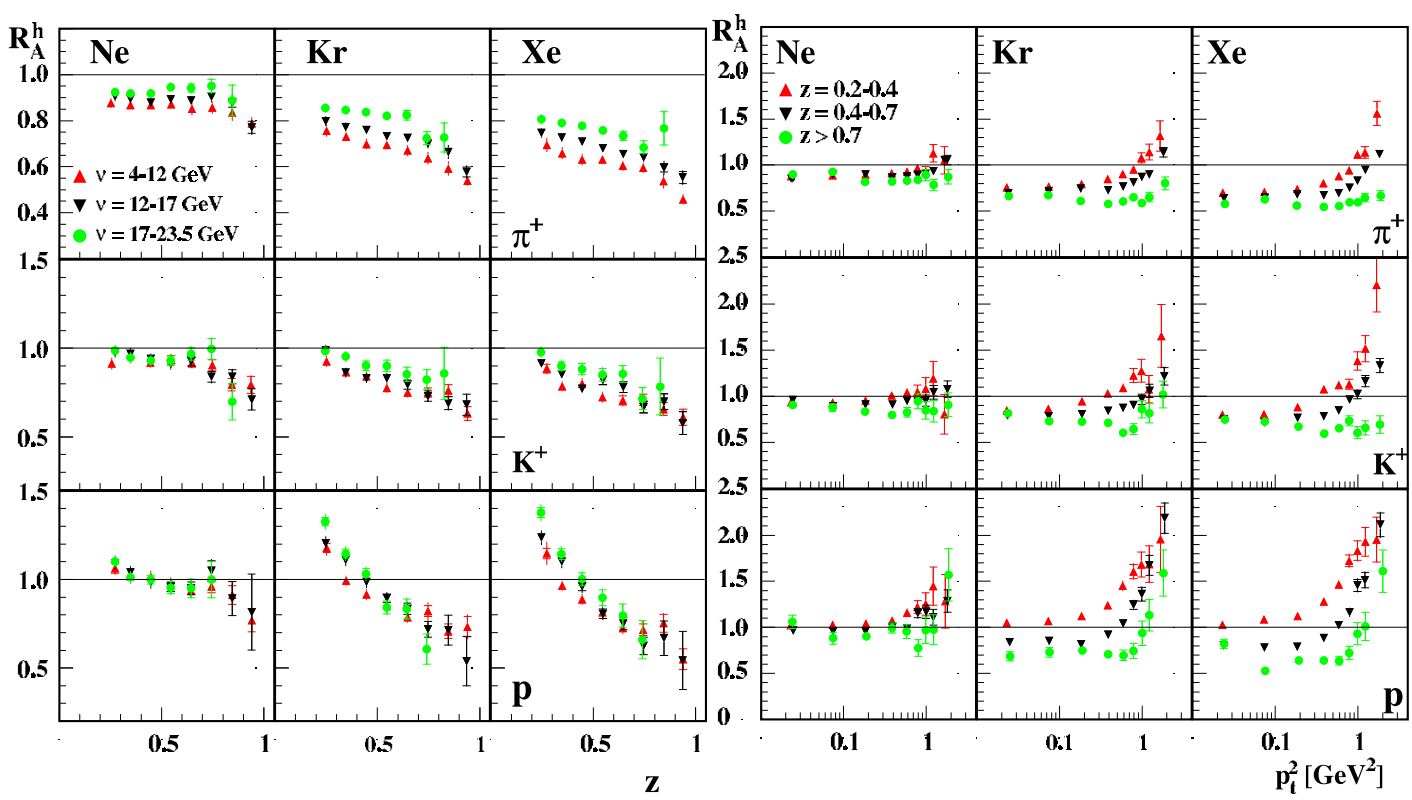

Fig. 2. Positive pion (top), positive kaon (middle), and proton (bottom) multiplicity ratios in bins of $(z, v)$ (left main panel) and $\left(P_{h \perp}^{2}, z\right)$ (right main panel), with $P_{h \perp}^{2}$ here labeled as $p_{t}^{2}$, for neon (left), krypton (middle), and xenon (right) targets. Statistical uncertainties are represented by the inner error bars; systematic uncertainties are represented by the outer error bars.

Similar considerations can also explain the observed transverse-momentum distributions from figure 1 . The transverse hadron momentum reflects both intrinsic transverse momentum of the interacting quark inside the nucleon and the transverse momentum it acquires during the fragmentation process. As can be seen, negative kaons exhibit a broader multiplicity distribution than positive kaons and pions. This can reflect a more complicated hadronization process for negative kaons. Considering for example the LUND model, which models the fragmentation process in terms of string breaking, unfavored fragmentation is characterized by at least one more string break in comparison with favored fragmentation, resulting in a broader distribution of the transverse momentum acquired in the fragmentation process.

\section{Hadronization in nuclei}

Ratios of charge-separated pion, proton, and kaon multiplicities measured on a neon, krypton, and xenon target and multiplicities measured on a deuterium target were extracted in bins of $(v, z),(z, v)$, $\left(P_{h \perp}^{2}, z\right)$, and $\left(z, P_{h \perp}^{2}\right)$ [7], with $v$, in the laboratory frame, the virtual-photon energy. In this notation, a fine binning is used for the first kinematic variable, while for the second variable a coarser binning is used. For the extraction of the multiplicity ratios, no correction for QED radiative effects, acceptance, and finite detector resolution is applied since most of these e ffects cancel when considering ratios.

Multiplicity ratios provide information on the time and length scales of hadron production. When scattering leptons off a large target nucleus, there is a higher probability for the struck quark and subsequently formed hadron to interact with the surrounding nuclear medium, and thus a larger attenuation for hadron production might be expected, in comparison with production on light nuclei. Comparing multiplicity ratios for various types of target nuclei can hence provide information on the length and time scales of the production of hadrons.

Multiplicity ratios, $R_{A}^{h}$, of positive pions, positive kaons, and protons for neon, krypton, and xenon targets in bins of $(z, v)$ and $\left(P_{h \perp}^{2}, z\right)$ are shown in figure 2. On the left main panel, a slight change in dependence on $z$ with varying $v$ is seen for positive pions. Both for pions and kaons, an increase 

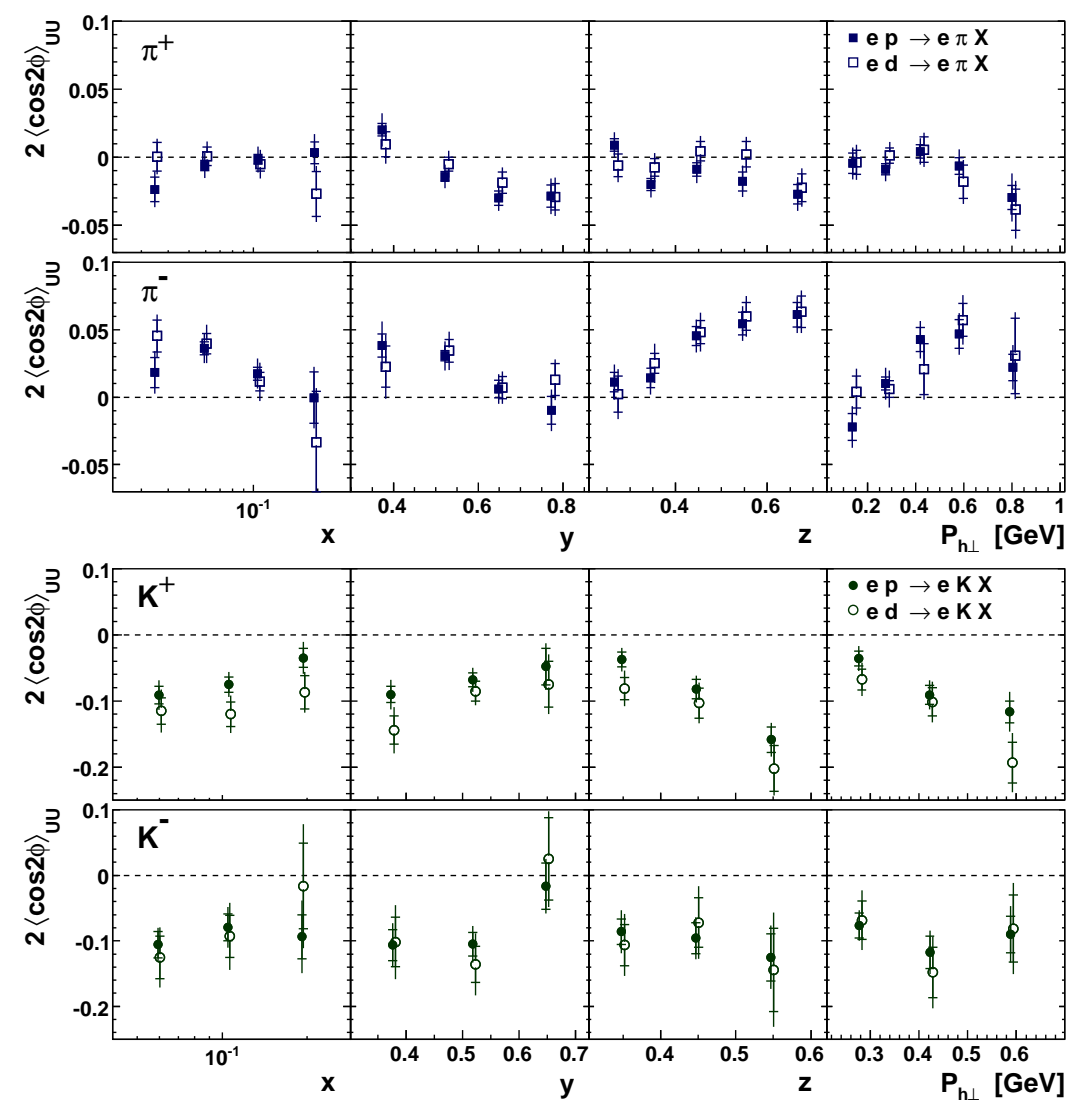

Fig. 3. Charged-separated $\cos 2 \phi$ moments for pions (upper two panels) and kaons (lower two panels) versus $x, y$, $z$, and $P_{h \perp}$ for protons (filled symbols) and deuterons (open symbols). Statistical uncertainties are represented by the inner error bars; systematic uncertainties are represented by the outer error bars.

in attenuation with increasing $z$ is observed. This effect is enhanced with larger target mass number. For protons, however, the multiplicity ratios exceed unity at low $z$ values. This can be attributed to final-state interactions: protons constituting the nuclear target can through interaction with a hadron (in formation) be ejected out of the nuclear target. These protons, on average, have low energy. In contrast, high-energy protons originate from the hadronization process.

The right main panel of figure 2 exhibits the behavior of $R_{A}^{h}$ as a function of $P_{h \perp}^{2}$. For pions and kaons, the multiplicity ratio rises with increasing $P_{h \perp}^{2}$ at low values of $z$, exceeding unity at large values of $P_{h \perp}^{2}$. This can be attributed to a broadening in the $P_{h \perp}^{2}$ distribution, due to the interaction of the struck quark or formed hadron with the nuclear medium. In contrast, at high $z$ values, in the limit of $z \rightarrow 1$, there is no room for rescattering, since the created hadron is not allowed to have lost energy. Again for protons, a different behavior is observed, consistent with large final-state interactions.

\section{Azimuthal distribution of charge-separated identified hadrons}

When considering the non-collinear semi-inclusive DIS cross section, two additional structure functions appear in comparison with the collinear cross section. One of these appears at leading twist, with a $\cos (2 \phi)$ modulation, whereas the other appears at sub-leading twist, with a $\cos (\phi)$ modulation. Here the angle $\phi$ is the angle between the lepton-scattering plane, i.e., the plane defined by the threemomenta of the incoming and scattered lepton, and the hadron-production plane, defined previously. 
The $\cos (2 \phi)$ term is a convolution of the Boer-Mulders distribution function and the Collins fragmentation function. The sub-leading twist structure function contains this same contribution together with a term related to the Cahn effect $[8,9]$ as well as quark-gluon-quark correlations.

The corresponding $\cos (\phi)$ and $\cos (2 \phi)$ moments were extracted from data collected on a hydrogen and deuterium target, using a fully differential unfolding procedure in $x, y, z$, and $P_{h \perp}$ in order to eliminate moments induced by higher-order QED effects or detector acceptance [6]. Here $y$ represents, in the target-rest frame, the fractional virtual-photon energy with respect to the beam-lepton energy.

In figure 3 the extracted $\cos (2 \phi)$ moments projected in bins of $x, y, z$, and $P_{h \perp}$ are presented for $\pi^{+}$(first panel from top), $\pi^{-}$(second panel from top), $K^{+}$(third panel from top), and $K^{-}$(fourth panel from top) for data collected on proton (filled symbols) and deuterium (open symbols). As can be seen, the results for the pion $\cos (2 \phi)$ moments for data collected on hydrogen and for data collected on deuterium are compatible. This hints at similar Boer-Mulders distributions for $u$-quarks and $d$-quarks. On the other hand, positive moments for $\pi^{-}$and small but negative moments for $\pi^{+}$are observed. This can be explained by a favored Collins fragmentation function (e.g., $u \rightarrow \pi^{+}$) with equal magnitude but opposite sign compared to the unfavored Collins fragmentation function (e.g., $u \rightarrow \pi^{-}$).

For $K^{+}$, the observed moment is negative, which, e.g., in the framework of the Artru model [10], can be explained by equal-sign Collins fragmentation functions for $u \rightarrow \pi^{+}$and $u \rightarrow K^{+}$. However, also a contribution from sea quarks can play a role in the observed $K^{+}$moment, which is of larger magnitude than the $\pi^{+}$moment. Contrary to negative pions, negative kaons have a $\cos (2 \phi)$ moment of negative sign, and in addition of equal magnitude as that of positive kaons. Based on $u$-quark dominance, this would signify that the Collins fragmentation functions for fragmentation of a $u$-quark into a kaon is insensitive to the kaon charge. However, also here, sea quarks can play a significant role.

\section{Single-spin asymmetries off transversely polarized protons}

Single-spin asymmetries measured from a transversely polarized proton show characteristic angular modulations. Each of the corresponding azimuthal amplitudes is related to convolutions of different distribution and fragmentation functions. The amplitude of the $\sin \left(\phi+\phi_{S}\right)$ modulation, with $\phi_{S}$ the azimuthal angle of the transverse component of the target spin with respect to the lepton scattering plane and about the virtual-photon direction, is interpreted as the convolution of the transversity distribution and the Collins fragmentation function. The amplitude of the $\sin \left(\phi-\phi_{S}\right)$ modulation is proportional to the convolution of the Sivers distribution function and the spin-independent fragmentation function. The former amplitude is referred to as the Collins amplitude; the latter as the Sivers amplitude. These amplitudes were extracted from a transversely polarized hydrogen target [11,12], and are presented in figure 5 as a function of $x, z$, and $P_{h \perp}$ for charged and neutral pions, and for charged kaons.

The Collins amplitudes, shown on the left panel of figure 5, rise with increasing $x$ and $z$ for $\pi^{+}, \pi^{-}$, and $K^{+}$. They are positive for $\pi^{+}$and $K^{+}$, and negative for $\pi^{-}$. The large amplitude for $\pi^{-}$, but with opposite sign with respect to the $\pi^{+}$amplitude, again hints at a Collins fragmentation function with opposite sign but equal magnitude for favored and unfavored fragmentation, as also indicated by the results shown in the previous section. The Collins amplitude for $K^{+}$is larger than for $\pi^{+}$, which again can be explained by a larger Collins fragmentation function for $u \rightarrow K^{+}$than for $u \rightarrow \pi^{+}$, in case of $u$-quark dominance. The vanishing $K^{-}$amplitude might be attributed to the non-negligible role of sea quarks, since for sea quarks the transversity distribution is expected to be small. The comparison of the results for $\pi^{0}, \pi^{+}$, and $\pi^{-}$shows that isospin symmetry is fulfilled.

The Sivers amplitudes are presented on the right panel of figure 5 for $\pi^{+}, \pi^{-}, \pi^{0}, K^{+}$, and $K^{-}$. The bottom panel also shows the Sivers amplitude for the $\pi^{+}-\pi^{-}$difference. The latter provides access to the valence-quark Sivers distribution and in addition suppresses contributions from vector-meson production. The Sivers $\pi^{+}$amplitude is significantly positive, rising with $z$, and with $P_{h \perp}$ at low values of $P_{h \perp}$, after which it reaches a plateau at higher $P_{h \perp}$ values. Assuming $u$-quark dominance, this positive amplitude corresponds to a negative $u$-quark Sivers distribution function. The $\pi^{-}$amplitude is consistent with zero, which can be attributed to canceling contributions from the Sivers $u$ - and $d$-quark distributions, whereas also here for the $\pi^{0}$ amplitude, isospin symmetry is fulfilled. The positive piondifference amplitude hints either at a valence $d$-quark distribution much larger than the valence $u$-quark 

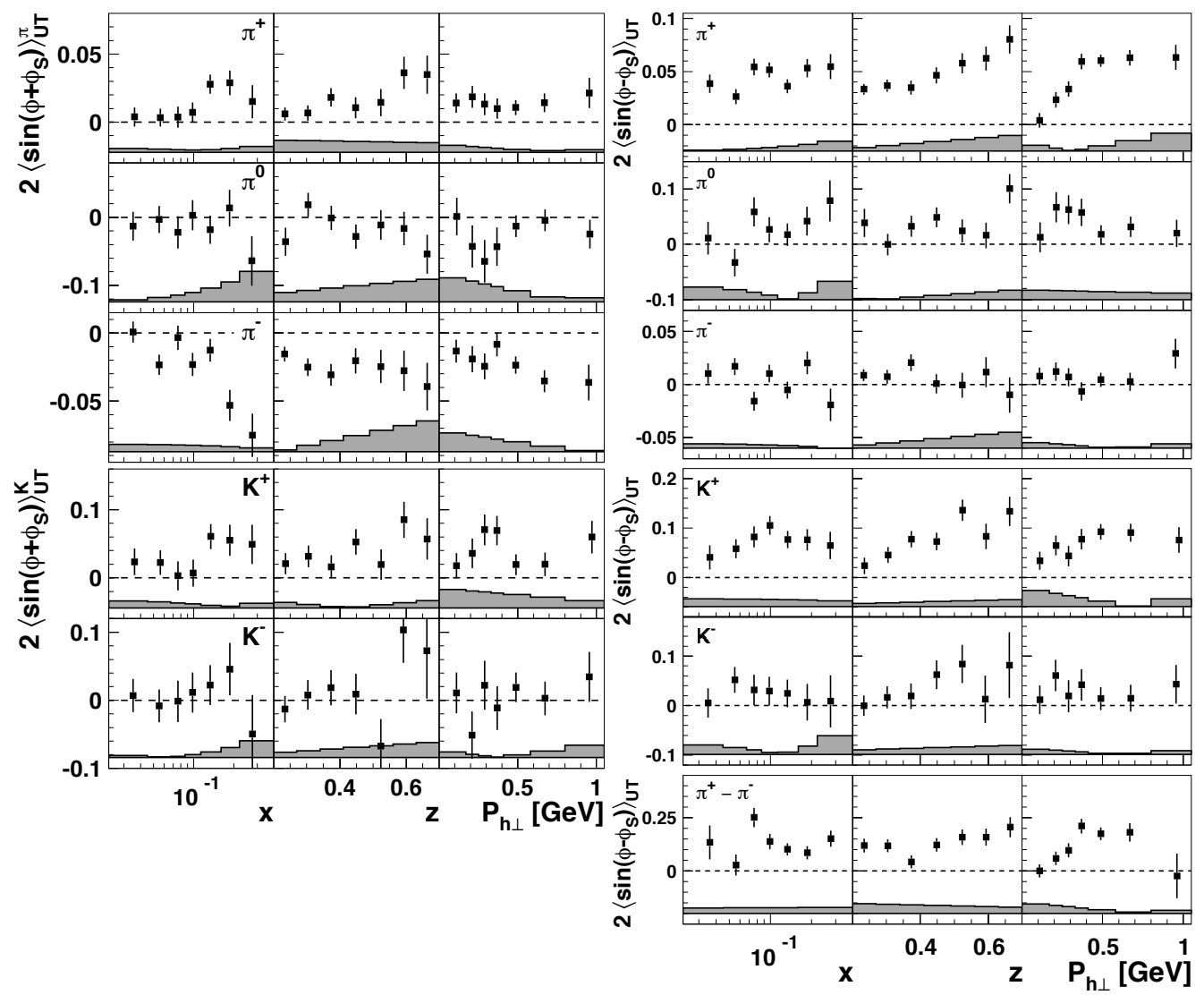

Fig. 4. Collins amplitudes (left panel) and Sivers amplitudes (right panel) as a function of $x, z$, and $P_{h \perp}$ for charged and neutral pions, and for charged kaons. Statistical uncertainties are represented by the error bars; systematic uncertainties are represented by the error bands.

distribution, or more probable, at a large negative valence $u$-quark Sivers distribution. The $K^{+}$Sivers amplitude shows a similar kinematic dependence as the $\pi^{+}$amplitude, but has a larger magnitude. This can hint at a non-trivial role of sea quarks. The $K^{-}$amplitude is also observed to be (slightly) positive.

\section{References}

1. J.C. Collins, Nucl. Phys. B 396 (1993) 161.

2. D. Boer and P. J. Mulders, Phys. Rev. D 57 (1998) 5780.

3. D. W. Sivers, Phys. Rev. D 41 (1990) 83.

4. J. P. Ralston and D. E. Soper, Nucl. Phys. B 152 (1979) 109.

5. S. J. Brodsky, D. S. Hwang, and I. Schmidt, Phys. Lett. B 530 (2002) 99.

6. A. Airapetian et al. [HERMES Collaboration], submitted to Phys. Rev. D, arXiv:1204.4161.

7. A. Airapetian et al., Eur. Phys. J. A 47 (2011) 113.

8. R. N. Cahn, Phys. Lett. B 78 (1978) 269.

9. R. N. Cahn, Phys. Rev. D 40 (1989) 3107.

10. X. Artru, J. Czyzewski, and H. Yabuki, Z. Phys. C 73 (1997) 527.

11. A. Airapetian et al., Phys. Rev. Lett. 103 (2009) 152002.

12. A. Airapetian et al., Phys. Lett. B 693 (2010) 11. 